\title{
CONGRUENCE AND DIMENSION OF NON-SEPARABLE METRIC SPACES
}

\author{
YASUNAO HATTORI \\ (Communicated by Dennis Burke) \\ Dedicated to Professor Yukihiro Kodama on his 60th birthday
}

\begin{abstract}
In this paper, we prove that, if a metrizable space $\mathbf{X}$ has an admissible metric such that $\mathbf{X}$ has no two distinct congruent subsets of cardinality 3 , then ind $\mathbf{X} \leq 1$. We also show that if a non-empty metrizable space $\mathbf{X}$ has an admissible star-rigid metric, then ind $\mathbf{X}=0$. The latter answers a question of L. Janos and H. Martin [3].
\end{abstract}

In the present note, we investigate a relation between certain special metrics and dimensions of non-separable metrizable spaces. For a metric space $(X, d)$, a point $x \in \mathbf{X}$ and a positive real number $\varepsilon$, we denote $U(x ; \varepsilon)=\{y \in$ $\mathbf{X}: d(x, y)<\varepsilon\}$ and $H(x ; \varepsilon)=\{y \in \mathbf{X}: d(x, y)=\varepsilon\}$. Recall from [2] that two subsets $\mathbf{A}$ and $\mathbf{B}$ of a metric space $(\mathbf{X}, d)$ are called congruent if there exists a bijection $f$ from A onto B such that $d(a, b)=d(f(a), f(b))$ for every $a$, $b \in \mathbf{A}$. In [1], Janos proved that a non-empty separable metrizable space $\mathbf{X}$ is zero-dimensional if, and only if, $\mathbf{X}$ has an admissible metric $d$ such that $\mathbf{X}$ contains no two distinct congruent subsets of cardinality 2 . Recently, he explored the case where the cardinality of the subsets is 3 in [2]. In particular, he showed that a locally compact, separable metrizable space $\mathbf{X}$ is at most onedimensional if $\mathbf{X}$ has an admissible metric $d$ satisfies the following condition:

(*) $\quad \mathbf{X}$ has no two distinct subsets of cardinality 3 that are congruent relative to $d$.

On the other hand, there exists a non-separable metric space $(\mathbf{X}, d)$ that satisfies the condition $(*)$ (see the example later in this paper). Thus, the following theorem improves the result of Janos.

Theorem 1. If the metrizable space $\mathbf{X}$ has an admissible metric satisfying the condition $(*)$, then ind $\mathbf{X} \leq 1$.

Received by the editors April 11, 1989. The contents of this paper have been presented to the meeting of the Mathematical Society of Japan at Nihon University, April 2, 1989.

1980 Mathematics Subject Classification (1985 Revision). Primary 54F45, 54F35; Secondary 54E40.

Key words and phrases. Metric spaces, congruence, small inductive dimension, star-rigid metric.

This research was supported in part by Grand-in-Aid for Scientific Research (No. 63740044), Ministry of Education, Science and Culture of Japan. 
Proof. Let $d$ be an admissible metric on $\mathbf{X}$ that satisfies the condition (*). Let $x \in \mathbf{X}$ and $\varepsilon>0$. It suffices to show that ind $H(x ; \varepsilon) \leq 0$. To show it, let $y \in H(x ; \varepsilon)$ and $\delta>0$. By the condition $(*)$, it follows that the cardinality of the set $H(x ; \varepsilon) \cap H(y ; \delta)$ is at most one. First, we suppose that $H(x ; \varepsilon) \cap H(y ; \delta)=\varnothing$. It is obvious that $V(y)=H(x ; \varepsilon) \cap U(y ; \delta)$ is an open-and-closed neighborhood of $y$ in $H(x ; \varepsilon)$.

Next, we suppose that $H(x ; \varepsilon) \cap H(y ; \delta)=\{z\}$. We put $G=\{w \in$ $\mathbf{X}: d(y, w)<d(z, w)\}$ and $V(y)=G \cap H(x ; \varepsilon) \cap U(y ; \delta)$. To show that the open neighborhood $V(y)$ of $y$ in $H(x ; \varepsilon)$ is closed in $H(x ; \varepsilon)$, let $w \in$ $H(x ; \varepsilon)-V(y)$. If $w \notin G$, the condition $(*)$ implies $d(y, w)>d(z, w)$. Then let $\delta^{\prime}=d(y, w)-d(z, w)>0$. It is easy to show that $G \cap U\left(w ; \delta^{\prime} / 2\right)=$ $\varnothing$. Hence $U\left(w, \delta^{\prime} / 2\right) \cap V(y)=\varnothing$. If $w \in G$, then $d(y, w) \geq \delta$.

Suppose that $d(y, w)=\delta$. Then $w \in H(x ; \varepsilon) \cap H(y ; \delta)$. On the other hand, $z \neq w$ and $z \in H(x ; \varepsilon) \cap H(y ; \delta)$. Hence $|H(x ; \varepsilon) \cap H(y ; \delta)| \geq 2$; this is a contradiction. Therefore, $d(y, w)>\delta$.

We put $\delta^{\prime \prime}=d(y, w)-\delta>0$. It follows that $U\left(w ; \delta^{\prime \prime}\right) \cap U(y ; \delta)=\varnothing$. Hence $U\left(w ; \delta^{\prime \prime}\right) \cap V(y)=\varnothing$. Thus $V(y)$ is closed in $H(x ; \varepsilon)$. This completes the proof.

The following example shows that the condition $(*)$ does not imply the separability of spaces.

Example. Well-order the set of all real numbers in the half-open interval $(0,1]$ as $\left\{t_{\alpha}: \alpha \in A\right\}$, where $A$ denotes the set of all ordinal numbers less than the cardinal of the continuum $c$. Let $\mathbf{S}(A)$ denote the star-space with an index set $A$ (see $[4, \mathrm{p} .111]$ for the definition of the star-spaces). Let $\rho$ be the metric on $\mathbf{S}(A)$ defined as

$$
\rho((x, \alpha),(y, \beta))= \begin{cases}|x-y|, & \text { if } \alpha=\beta, \\ x+y, & \text { if } \alpha \neq \beta,\end{cases}
$$

for each $(x, \alpha),(y, \beta) \in \mathbf{S}(A)$.

Let $\mathbf{X}=\left\{\left(t_{\alpha}, \alpha\right): \alpha \in A\right\}$ be the subspace of $\mathbf{S}(A)$. It is easy to show that the metric space $(\mathbf{X}, \rho)$ satisfies the condition $(*)$, but the weight of $(\mathbf{X}, \rho)$ is equal to $c$.

In [3], Janos and Martin studied the relations between the star-rigid metric and zero-dimensionality of separable metrizable spaces. Recall from [3] that a metric $d$ on a space $\mathbf{X}$ is called star-rigid if, for every points $x, y$ and $z$ of $\mathbf{X}$ with $y \neq z, d(x, y) \neq d(x, z)$. Janos and Martin proved that for a non-empty separable metrizable space $\mathbf{X}, \mathbf{X}$ is zero-dimensional if, and only if, $\mathbf{X}$ has an admissible totally-bounded star-rigid metric, and they asked whether for every non-empty metrizable (not necessarily separable) space $\mathbf{X}$, ind $\mathbf{X}=0$ holds, if $\mathbf{X}$ has an admissible star-rigid metric [3, Question 2]. An argument similar to the proof of Theorem 1 shows the following theorem which answers the question. 
Theorem 2. If a non-empty metrizable space $\mathbf{X}$ has an admissible star-rigid metric, then ind $\mathbf{X}=0$.

Proof. Let $d$ be an admissible metric on $\mathbf{X}, x \in \mathbf{X}$ and $\varepsilon>0$. If $H(x ; \varepsilon)=$ $\varnothing$, then $U(x ; \varepsilon)$ is an open-and-closed neighborhood of $x$.

Suppose that $H(x ; \varepsilon)=\varnothing$. Since $d$ is star-rigid, $|H(x ; \varepsilon)|=1$. Let $\{z\}=$ $H(x ; \varepsilon)$ and $G=\{w \in \mathbf{X}: d(x, w)<d(z, w)\}$. Since $d$ is star-rigid, $G$ is an open-and-closed set of $\mathbf{X}$. We put $V=U(x ; \varepsilon) \cap G$. It can be seen that $V$ is an open-and-closed neighborhood of $x$ by an argument similar to the proof of Theorem 1. Hence ind $\mathbf{X}=0$.

Remark. It is obvious that ind $\mathbf{X}=0$ for the metric space $(\mathbf{X}, \rho)$ mentioned in the example. Therefore, we can ask the following question: Does there exist a non-separable metric space $(\mathbf{X}, d)$ such that ind $\mathbf{X}=1$ and the condition $(*)$ is satisfied?

\section{REFERENCES}

1. L. Janos, A metric characterization of zero-dimensional spaces, Proc. Amer. Math. Soc. 31 (1972), 268-270.

2. __ Congruence and one-dimensionality of metric spaces, Proc. Amer. Math. Soc. 103 (1988), 1268-1270.

3. L. Janos and H. Martin, Metric characterizations of dimension for separable metric spaces, Proc. Amer. Math. Soc. 70 (1978), 209-212.

4. J. Nagata, Modern general topology, 2nd ed., North-Holland, Amsterdam, 1985.

Department of Mathematics, Faculty of Education, Yamaguchi University, YAMAGUCHI 753 JAPAN 